\title{
The Planck Catalogue of Galactic Cold Clumps : PGCC
}

\author{
L. Montier ${ }^{1,2}$ \\ on behalf of the Planck collaboration \\ ${ }^{1}$ CNRS, IRAP, 9 Av. colonel Roche, BP 44346, F-31028 Toulouse cedex 4, France \\ ${ }^{2}$ Université de Toulouse, UPS-OMP, IRAP, F-31028 Toulouse cedex 4, France
}

\begin{abstract}
The Planck satellite has provided an unprecedented view of the submm sky, allowing us to search for the dust emission of Galactic cold sources. Combining Planck-HFI all-sky maps in the high frequency channels with the IRAS map at 100um, we built the Planck catalogue of Galactic Cold Clumps (PGCC, Planck 2015 results. XXVIII), counting 13188 sources distributed over the whole sky, and following mainly the Galactic structures at low and intermediate latitudes. This is the first all-sky catalogue of Galactic cold sources obtained with a single instrument at this resolution and sensitivity, which opens a new window on star-formation processes in our Galaxy.
\end{abstract}

Keywords. submillimeter, ISM: clouds, ISM: structure, stars: formation

\section{Introduction}

During the past decade, new insights into the study of cold sources have been provided by sophisticated numerical modelling and by the development of sensitive millimetre and submillimetre detectors, operating both from space and from the ground, and with either imaging or spectroscopic capabilities. By combining the highest frequency channels of the Planck survey $(353-857 \mathrm{GHz}, 850-350 \mu \mathrm{m})$ with the far-infrared IRAS data, and by applying a dedicated source detection method, which leverages on the spectral signature of cold sources, we can obtain an all-sky census of the coldest Galactic objects. In particular, the method of Montier et al (2010) makes it possible to separate cold and warm dust emission components, and to derive the physical properties (flux density, size of the emitting region, temperature) of the cold component. Furthermore, Planck has provided the first uniform submillimetre surveys that cover both the Galactic plane and regions at intermediate and high latitudes, which allows us to expand the physical parameter space probed by the previously known cold sources.

As part of the first Planck data release, the sample of the most robust Planck detections has already been delivered to the astronomical community, as the Early Cold Clump sample (ECC) included 915 Planck cold clumps (at $T<14 \mathrm{~K}$ ) distributed over the whole sky (Planck early results. VII, 2011, Planck early results. XXII, 2011 and Planck early results. XXIII, 2011). We present here the entire catalogue of 13188 cold sources, i.e., the Planck Catalogue of Galactic Cold Clumps (PGCC, Planck 2015 results. XXVIII), which has been delivered with the full Planck 2015 data release (Planck 2015 results. I).

\section{Overview}

The PGCC catalogue counts 13188 sources located over the whole sky (plus 54 sources located in the Small and Large Magellanic Clouds) and presenting a mean temperature of 13 and $14.5 \mathrm{~K}$. For 5574 of them, a distance estimate is provided, leading to a complete set of physical properties, including temperature, column density, mass, density and physical size. Hence about $85 \%$ of the PGCC sources are located below $1 \mathrm{kpc}$ from the sun, thus in the local neighbourhood. The column density estimates cover almost five orders of magnitude, starting from $6.8 \times 10^{18} \mathrm{~cm}^{-2}$ to very massive objects. Similarly the physical size of the PGCC sources ranges from $0.14 \mathrm{pc}$ to $30.6 \mathrm{pc}$, spanning a broad variety of objects, from cold cores to giant molecular clouds. 


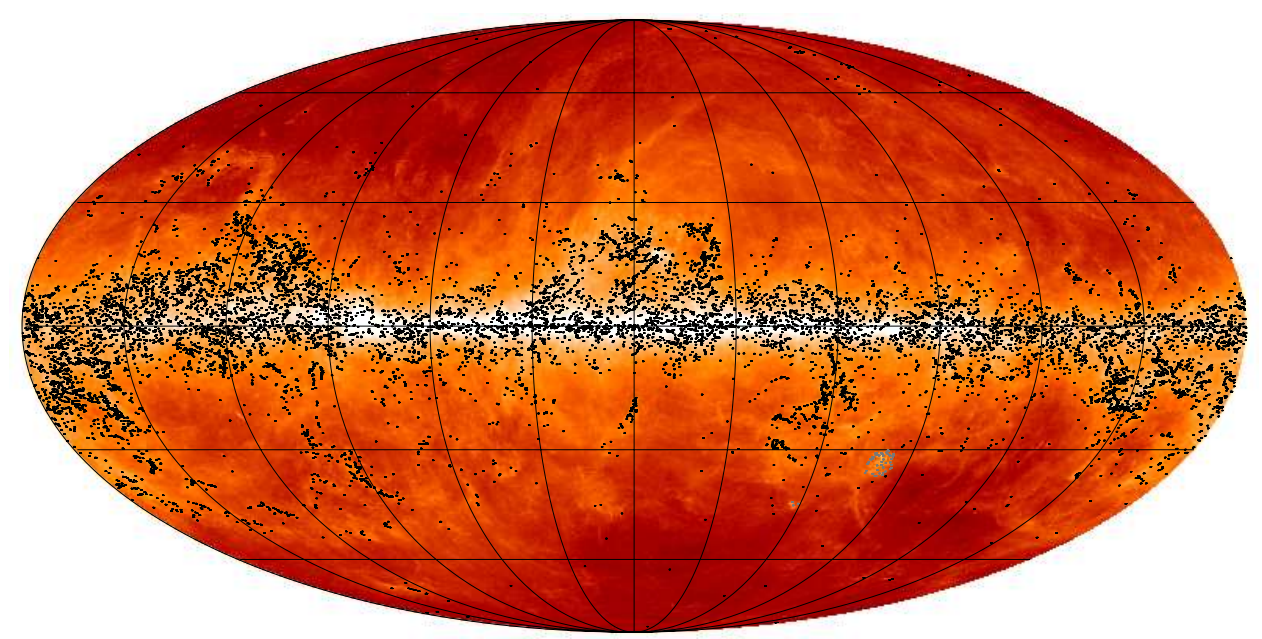

Figure 1. All-sky distribution of the PGCC sources, overlaid on the Planck $857 \mathrm{GHz}$ map.

The catalogue may be downloaded from the Planck Legacy Archive (www.cosmos.esa.int/ web/planck/pla), or from the MuFFInS platform (Multi Frequency Followp-up Inventory Service, https://muffins.irap.omp.eu).

\section{Implications}

The PGCC catalogue, covering the whole sky, hence probing wildly different environments, represents a real goldmine for investigations of the early phases of star formation. These include, but are not limited to: i) studies of the evolution from molecular clouds to cores and the influence of the local conditions; ii) analysis of the extreme cold sources, such as the most massive clumps or those located at relatively high latitude; iii) characterization of the dust emission law in dense regions and the role of the environment. A large follow-up of 315 PGCC sources has been performed with Herschel PACS \& SPIRE, leading to a series of original results about the evolution of star-formation in cold cores (Juvela et al, 2010, 2011, 2012, 2015a and 2015b).

\section{References}

Montier, L. A., Pelkonen, V., Juvela, M., Ristorcelli, I., \& Marshall, D. J., 2010, A\& A, 522, A83 Planck Collaboration, Planck early results. VII, 2011, A\&A, 536, A7

Planck Collaboration, Planck early results. XXII, 2011, A\&A, 536, A22

Planck Collaboration, Planck early results. XXIII, 2011, A\&SA, 536, A23

Planck Collaboration, Planck 2015 results. I, 2015, subm to $A \mathscr{E} A$, arXiv:1502.01582

Planck Collaboration, Planck 2015 results. XXVIII, 2015, A\& $A$ in press, arXiv:1502.01599

Juvela, M., Ristorcelli, I., Montier, L. et al.2010, AछA, 518, L93

Juvela, M., Ristorcelli, I., Pelkonen. V.-M. et al.2011, A\& A, 527, A111

Juvela, M., Ristorcelli, I., Pagani, L. et al.2012, A\&A, 541, A12

Juvela, M., Ristorcelli, I., Marshall, D. et al.2015, A\&A in press, arXiv:1501.07092

Juvela, M., Demyk, K., Doi, Y. et al.2015, AESA in press, arXiv:1509.08023 